Indem wieder darauf hingewiesen wird, daß die gemessenen Zahlenwerte in der späteren Veröffentlichung tabellarisch zusammengestellt werden, sind unsere Ergebnisse unter Vergleich mit der Kurve von Craig usw. in Abb. 2 graphisch dargestellt. Die einzelnen Meßpunkte entstammen dabei je zwei mit der gleichen Probe durchgeführten Meßreihen. Der Wasserstoffgehalt der entgasten Probe war gleich Null, derjenige der wasserstoffhaltigen betrug $21 \mathrm{~cm}^{3}$ pro $100 \mathrm{~g}$ Metall (bezogen auf $760 \mathrm{~mm} \mathrm{Hg}$ und $0^{\circ} \mathrm{C}$ ), entsprechend $4,56 \cdot 10^{-2}$ At. $\%$ H. Die $C_{p}$-Werte der letzteren Probe wurden auf das Atomgewicht des Magnesiums bezogen.

Abgesehen von geringfügigen Abweichungen im Temperaturgebiet zwischen 35 und $60^{\circ} \mathrm{K}$ decken sich unsere Meßpunkte betr. Reinmagnesium mit der Kurve von Craig bzw. Clusius und Vaughen. Aus dieser Übereinstimmung schließen wir zunächst auf die Richtigkeit unserer Meßergebnisse, halten damit indessen auch umgekehrt den Verlauf der $C_{p}$-T-Kurve des Reinmagnesiums für gesichert. In Übereinstimmung mit dem von Craig angegebenen Wert ergibt sich für den Standardwert der Entropie des Magnesiums $7,87 \mathrm{cal} / \mathrm{Gr}$-Atom.

Der Verlauf der $C_{p}$-T-Kurve des wasserstoffhaltigen Magnesiums deckt sich bis etwa $90{ }^{\circ} \mathrm{K}$ ebenfalls mit der Kurve von Craig bzw. unseren Werten für Reinmagne- sium. Oberhalb dieser Temperatur tritt indessen ein systematisches Abweichen zu höheren $C_{p}$-Werten in dem Sinne auf, daß die Abweichungen mit steigender Temperatur größer werden. Ein Vergleich dieser Kurve mit der von Eastman und Rodebush ermittelten, insbesondere im Hinblick auf die Temperatur des Beginnens und den Richtungssinn, sowie die zunehmende Größe der Abweichungen mit steigender Temperatur, legt die Vermutung nahe, daß Eastman und Rodebush ebenfalls wasserstoffhaltiges Magnesium verwendet haben. Diese Annahme erscheint um so gerechtfertigter, als zu Zeiten dieser Messungen die in Metallen gelösten Gase noch nicht im heutigen Umfange in den Blickpunkt der Physiker gerückt waren.

Indem wir eine genauere Diskussion der Verhältnisse der angekündigten späteren Veröffentlichung vorbehalten, möchten wir nur noch darauf hinweisen, da $\beta$ ein ähnlicher abweichender Kurvenverlauf zwischen reinem und wasserstoffhaltigem Palladium kürzlich von EıcheNAUER und $\mathrm{S}_{\text {CHÄFER }}{ }^{8}$ mitgeteilt worden ist. Die von diesen Autoren angegebenen Kurven reichen zwar nur bis zu etwa $60{ }^{\circ} \mathrm{K}$ herab, lassen indessen deutlich werden, da $\beta$ die Temperatur beginnender Abweichung ebenfalls bei ungefähr $80-90{ }^{\circ} \mathrm{K}$ liegt.

8 W.Eichenauer u. L.Schäfer, Z. Naturforschg. 11 a, 955[1956].

\section{Elektrodenvorgänge im System Siliciumeinkristall/Natronlauge}

\author{
Von M. SEIPT \\ Institut für Physikalische Chemie und Elektrochemie \\ der Technischen Hochschule Karlsruhe \\ (Z. Naturforschg. 14 a, 926 -928 [1959]; eingeg. am 19. August 1959)
}

Das System Silicium/Elektrolyt sei durch das Schema

$$
\mathrm{H} / \mathrm{RS} / \mathrm{S} / \mathrm{IS} / \mathrm{Lö}
$$

charakterisiert. H bedeutet das neutrale Halbleiterinnere, RS die Halbleiterrandschicht, S die Siliciumoberfläche, IS die „Ionenschicht“, welche das lösungsseitige Analogon zur Randschicht ist, Lö das neutrale Innere des Elektrolyten. Wenn die Siliciumoberfläche eine direkte Wechselwirkung zwischen Rand- und Ionenschicht verhindert bzw. genügend klein hält, dann läßt sich das Schema (1) in zwei Teilsysteme zerlegen,

$$
\mathrm{H} / \mathrm{RS} / \mathrm{S} \text { und S / IS / Lö, }
$$

welche hinsichtlich ihrer elektrischen Eigenschaften hintereinandergeschaltet sind und es ergibt sich die Aufgabe, die Verteilung des Spannungsabfalles auf die beiden Teilsysteme zu ermitteln.

\section{A. Strombelastete Elektrode}

Bei nicht zu geringer Strombelastung überwiegt der Widerstand eines Teilsystemes, und dieses bestimmt das Verhalten des Gesamtsystemes. Wenn die Siliciumelektrode in Sperrichtung gepolt ist $(\mathrm{n}-\mathrm{Typ}=$ Anode, $\mathrm{p}$-Typ
= Kathode), dann ist das Teilsystem H/RS / S hochohmig, der Spannungsabfall über der Randschicht (Randschichtüberspannung) ist sehr viel größer als die elektrochemische Überspannung. Umgekehrt dominiert die elektrochemische Überspannung bei Polung in Flußrichtung ( -Typ $=$ Kathode, p-Typ $=$ Anode $)$. Außerdem läßt sich die Sperrung einer sperrbelasteten Elektrode innerhalb eines gewissen Stromdichtebereiches bei Bestrahlung mit weißem Licht (innerer Photoeffekt) aufheben, so daß dann auch die elektrochemische Überspannung direkt gemessen wird (Abb. 1). Die kathodische Wasserstoffüberspannung befolgt die TAFEL-Gleichung

$$
\Delta u=a-b \lg |J|
$$

( $\Delta u$ Überspannung; $a, b$ Konstanten, $J$ Stromdichte). Der $b$-Wert beträgt $(0,95 \pm 0,1) \mathrm{V}$. Er liegt damit etwas tiefer als der theoretische Wert $1,12 \mathrm{~V}$. Vermutlich wird diese Erniedrigung durch einen z. Zt. noch unbekannten, depolarisierenden Elektrodenprozeß verursacht. Die anodischen Stromspannungskurven sind sämtlich um $+15,5 \mathrm{~V}$ verschoben (s. Abb. 1). Ursache davon ist eine Passivschicht auf der Siliciumoberfläche. Merkbare Abhängigkeit dieses Spannungsabfalles von der Stromdichte konnte nicht festgestellt werden. Aus dem Verlauf der "Sperrkennlinien“ der unbelichteten, sperrbelasteten Elektroden sieht man (Abb. 1), daß nur sehr unvollkommene Sperrung auftritt. Dies weist auf Prozesse in der Oberfläche und - wie auf Grund der beobachteten Zeiteffekte geschlossen wird - in der Randschicht hin, welche die Übergangswahrscheinlichkeit für Ladungsträger zwischen dem Valenz- und dem Leitfähigkeitsband mit zunehmender Feldstärke stark erhöhen. 


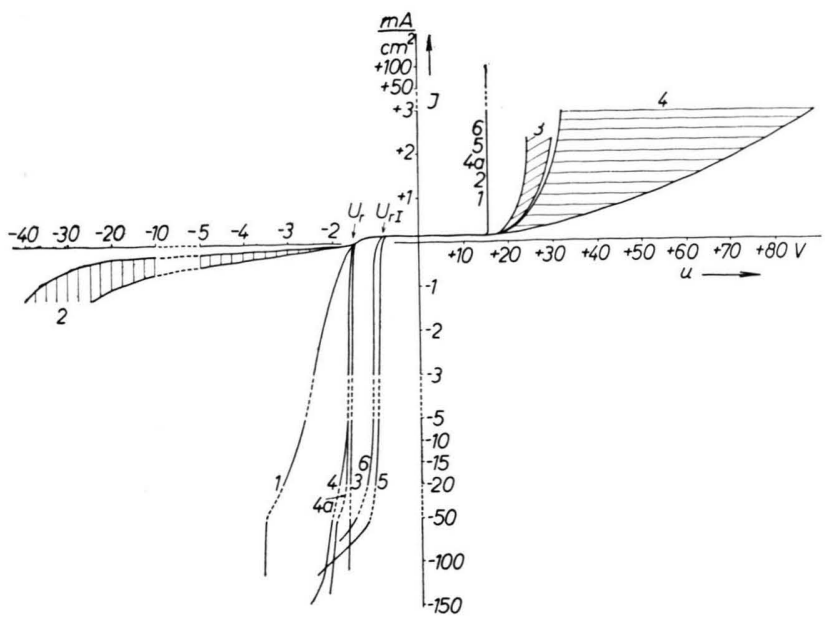

Abb. 1. Stationäre Gesamtstromspannungskurven.

1) p-Typ, $0,2 \Omega \cdot \mathrm{cm}$, unbelichtet;

2) p-Typ, $20 \Omega \cdot \mathrm{cm}$, unbelichtet;

3) n-Typ, $0,2 \Omega \cdot \mathrm{cm}$, unbelichtet;

4) n-Typ, $20 \Omega \cdot \mathrm{cm}$, unbelichtet;

4a) n-Typ, $20 \Omega \cdot \mathrm{cm}$, belichtet ;

5) p-Typ, $0,2 \Omega \cdot \mathrm{cm}$, belichtet;

6) p-Typ, $20 \Omega \cdot \mathrm{cm}$, belichtet;

$u_{\mathrm{r}}=$ Ruhepotential an unbelichteter Elektrode;

$u_{\mathrm{rI}}=$ Ruhepotential an belichteter Elektrode.

Es wird vermutet, daß atomarer Wasserstoff in die Randschicht eindiffundiert und dort zusätzliche Rekombinationszentren bildet.

\section{B. Photo-EMK an stromloser Elektrode}

Das Potential der stromlosen, unbelichteten Siliciumelektrode ist von der Dotierung des Siliciums und damit von dessen Leitungstyp unabhängig und hängt allein

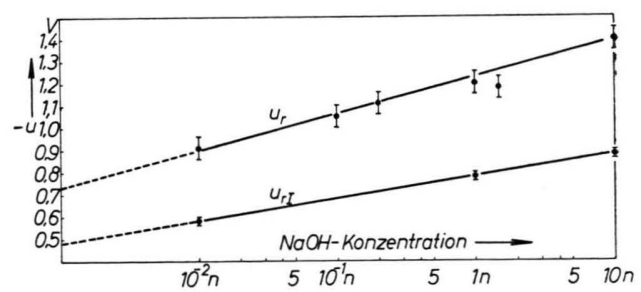

Abb. 2. Ruhepotential in Abhängigkeit der NaOH-Konzentration an einer p-Typ-Elektrode, $\varrho=0,2 \Omega \cdot \mathrm{cm}$.

Obere Kurve: unbelichtet; untere Kurve: belichtet.

von der Konzentration der Natronlauge ab. Diese $\mathrm{Ab}$ hängigkeit läßt sich bei $50{ }^{\circ} \mathrm{C}$ durch die Beziehung

$u_{\mathrm{r}}=-1,24-0,17 \lg c_{\mathrm{NaOH}}$
( $u_{\mathrm{r}}$ Ruhepotential, $c$ Konzentration der Natronlauge) wiedergeben (Abb. 2, obere Kurve). Bei Belichtung verhält sich die Elektrode wie ein Photo-Element mit hohem Innenwiderstand. Die entstehende Photo-EMK, $V_{\mathrm{Ph}}$, an unbelasteter Elektrode sollte deshalb im Grenzfalle hoher Belichtungsintensität entgegengesetzt gleich der Diffusionsspannung sein. Bei der verwendeten Belichtung änderte sich $V_{\mathrm{Ph}} \sim I^{0,05}$, so daß $V_{\mathrm{Ph}} \approx-V_{\mathrm{D}}$ angenommen werden kann. An der belichteten Elektrode (Abb. 2, untere Kurve) ergab sich

$$
V_{\mathrm{Ph}}=+0,46+0,7 \lg c_{\mathrm{NaOH}} \quad(\mathrm{V}) .
$$

Die Laugenkonzentration beeinflußt daher nicht nur die Korrosionsgeschwindigkeit des Siliciums, sondern auch die Diffusionsspannung und damit die Randkonzentration der Majoritätsträger. Für letztere gilt:

$$
\begin{array}{lll} 
& V_{\mathrm{D}}=(k T / e) \ln n_{\mathrm{H}} / n_{\mathrm{R}} & \text { (für n-Typ) } \\
\text { bzw. } & V_{\mathrm{D}}=(k T / e) \ln p_{\mathrm{H}} / p_{\mathrm{R}} & \text { (für } \mathrm{p}-\mathrm{Typ})
\end{array}
$$

[ $k$ Boltzmann-Konstante, $T$ abs. Temperatur, $e$ Elementarladung, $n, p$ Gleichgewichtskonzentration der Elektronen bzw. Löcher im Inneren $(\mathrm{H})$ bzw. am Rande (R) ].

Wie Abb. 3 zeigt, besteht zwischen $V_{\mathrm{D}}$ und $\ln n_{\mathrm{H}}$ jedoch kein lineares Verhältnis, wie es Gl. (6) verlangt

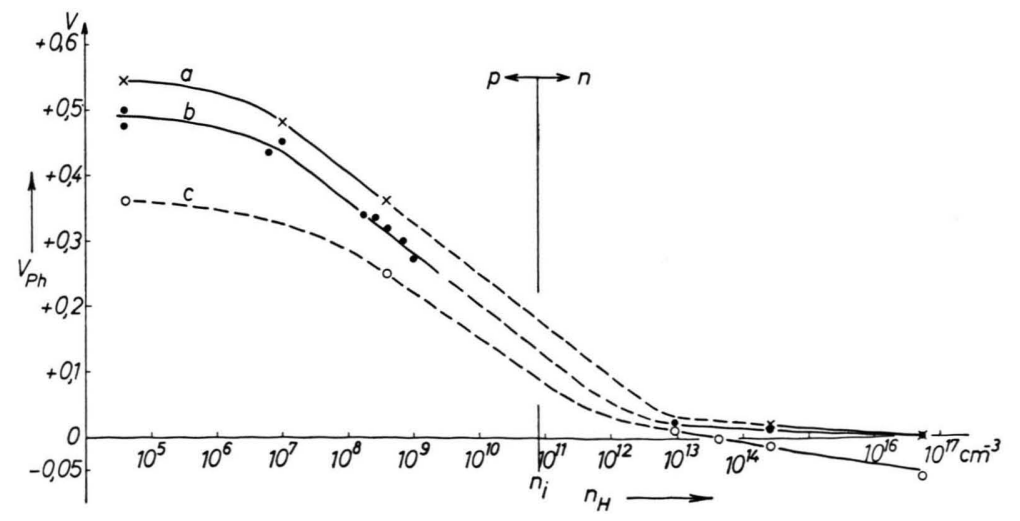

Abb. 3. Photo-EMK in Abhängigkeit der Gleichgewichtskonzentration der Elektronen: a) in $10-n$. $\mathrm{NaOH}, I=0$; b) in $10-n$. $\mathrm{NaOH}, I=136 I_{0} ;$ c) in $0,1-n \mathrm{NaOH}$, $I=136 I_{0} ; \quad I=$ Belichtungsintensität in willkürlichen Einheiten $I_{0} ; n_{\mathrm{i}}=$ Inversionsdichte. 


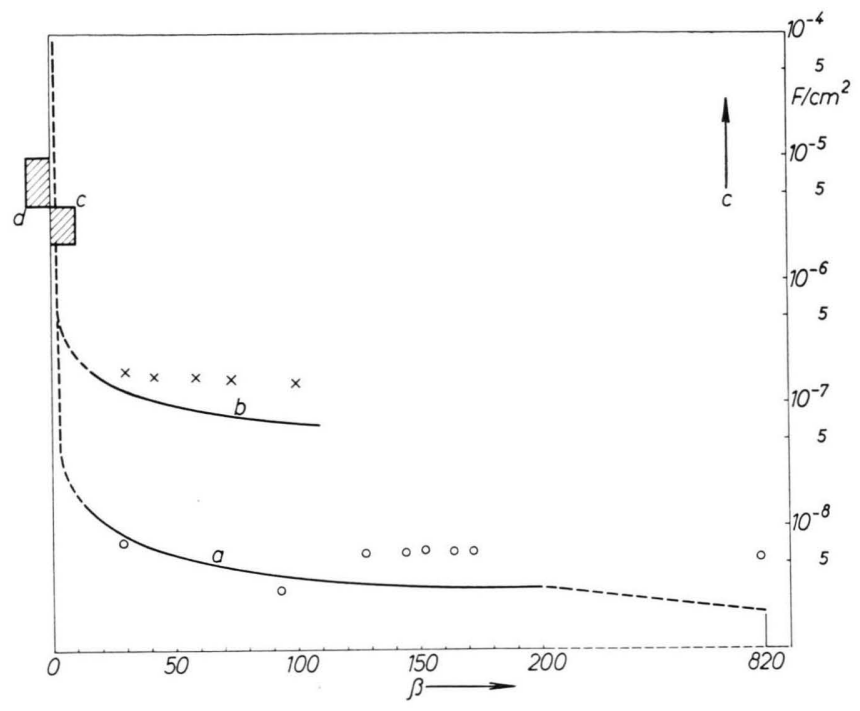

Abb. 4. Phasengrenzkapazität als Funktion der Randschichtspannung. a) $20 \Omega \cdot \mathrm{cm}$ p-Typ, $I=0$; b) $0,2 \Omega \cdot \mathrm{cm}$ p-Typ, $I=0$; c) $20 \Omega \cdot \mathrm{cm}$ p-Typ, $I=136 I_{0} ;$ d) $20 \Omega \cdot \mathrm{cm}$ n-Typ. Ausgezogene Kurven: theoretischer Verlauf nach Sсноттку [Gl. (7)].

-. höchstens in einem kleinen, hochohmigen Bereich könnte Gl. (6) annähernd erfüllt sein -, sondern es zeigt sich eine Sätigung mit zunehmender n- bzw. pDotierung. Die höchste gemessene Photo-EMK betrug $0,6 \mathrm{~V}$, das ist etwas mehr als die Hälfte des nach Gl. (6) für die beiden $0,2-\mathrm{Ohm} \cdot \mathrm{cm}$-Elektroden zu erwartenden Wertes. Da zu geringe Belichtungsintensität als mögliche Erklärung ausfällt (s. o.), muß es sich um eine echte Abweichung der Diffusionsspannung von Gl. (6) handeln. Diese kann nur dadurch hervorgerufen werden, daß an der Oberfläche zusätzlich örtlich fixierte Ladungen entstehen oder verschwinden, d. h. es müssen im verbotenen Bereich zwischen Leitfähigkeits- und Valenzband liegende Oberflächenterme vorhanden sein, deren Ladungszustand eine Funktion ihrer Lage relativ zum Fermi-Niveau ist. Bis jetzt sind jedoch noch keine näheren Angaben möglich, ob es sich um Terme in nur einer energetischen Lage handelt oder ob Terme in verschiedenen energetischen Lagen auftreten.

\section{Kapazität der Elektrode}

Das den Schemata (2) entsprechende, elektrische Ersatzschaltbild besteht im einfachsten Falle aus zwei in Serie geschalteten „Verlustkondensatoren“, in denen die Kapazitäten sowie ihre Parallelwiderstände belastungsabhängig sind. Dominiert die Randschicht, dann läßt sich die Kapazität der Elektrode durch die von Sсноттку für den Halbleiter/Metallkontakt abgeleitete Beziehung

$$
C=\left(\varepsilon \varepsilon_{0} e / 2 \beta\right)^{1 / 2}\left(N_{\mathrm{A}} \beta / u+V_{\mathrm{D}}\right)^{1 / 2} \quad \text { (für p-Typ) (7) }
$$
( $\varepsilon$ Dielektrizitätskonstante des Siliciums, $\varepsilon_{0}$ Influenzkonstante, e Elementarladung, $N_{\mathrm{A}}$ Akzeptorendichte, $V_{\mathrm{D}}$ Diffusionsspannung, $u$ Randschichtüberspannung, $\beta=k T / e=0,028 \mathrm{~V}$ für $T=50{ }^{\circ} \mathrm{C}$ ) darstellen, solange $u+V_{\mathrm{D}}>\approx 10 \beta$ ist; für sehr kleine Spannungen $u+V_{\mathrm{D}}$ gilt die Beziehung (7) nicht mehr (Abb. 4). Tatsächlich dürfte die Gl. (7) sogar noch besser erfüllt sein wie es in Abb. 4 scheint, da die experimentellen Werte z. Tl. etwas zu groß sein können, keinesfalls jedoch zu klein. - Im Flußbereich, $u+V_{\mathrm{D}} \approx 0$, sollte die Kapazität der Randschicht groß gegen die der Doppelschicht werden, so daß deren Kapazität, die etwa $20 \mu \mathrm{F} / \mathrm{cm}^{2}$, gemessen würde. Wie aus Abb. 4 hervorgeht, traten bei Flußbelastung sowie bei intensiv belichteten, sperrbelasteten Elektroden kleinere Werte auf $\left(5-10 \mu \mathrm{F} / \mathrm{cm}^{2}\right.$ bzw. $\left.2-4 \mu \mathrm{F} / \mathrm{cm}^{2}\right)$. Es wird angenommen, daß auch in diesen Fällen die Randschichtkapazität erst etwa die Größenordnung derjenigen der Doppelschichtkapazität erreicht hat und noch nicht groß gegenüber letzterer ist. Das bedeutet, daß stets noch eine Diffusionsspannung von einigen $k T / e$ vorhanden gewesen sein muß; deren genaue Größe läßt sich jedoch aus diesen Messungen nicht bestimmen. 